\title{
Electromagnetic Interpretation of Fractional-Order Elements
}

\author{
Guishu Liang, Jiawei Hao*, Dongqing Shan \\ Department of Electric Engineering, North China Electric Power University, Baoding, China \\ Email: *ncepuhjw@163.com
}

How to cite this paper: Liang, G.S., Hao, J.W. and Shan, D.Q. (2017) Electromagnetic Interpretation of Fractional-Order Elements. Journal of Modern Physics, 8, 2209-2218. https://doi.org/10.4236/jmp.2017.814136

Received: November 8, 2017

Accepted: December 18, 2017

Published: December 21, 2017

Copyright $\odot 2017$ by authors and Scientific Research Publishing Inc. This work is licensed under the Creative Commons Attribution International License (CC BY 4.0).

http://creativecommons.org/licenses/by/4.0/

\begin{abstract}
Fractional circuits have attracted extensive attention of scholars and researchers for their superior performance and potential applications. Recently, the fundamentals of the conventional circuit theory were extended to include the new generalized elements and fractional-order elements. As is known to all, circuit theory is a limiting special case of electromagnetic field theory and the characterization of classical circuit elements can be given an elegant electromagnetic interpretation. In this paper, considering fractional-order time derivatives, an electromagnetic field interpretation of fractional-order elements: fractional-order inductor, fractional-order capacitor and fractional-order mutual inductor is presented, in terms of a quasi-static expansion of the fractional Maxwell's equations. It shows that fractional-order elements can also be interpreted as a fractional electromagnetic system. As the element order equals to 1 , the interpretation of fractional-order elements matches that of the classical circuit elements: $L, C$, and mutual inductor, respectively.
\end{abstract}

\section{Keywords}

Fractional Circuits, Fractional-Order Elements, Maxwell's Equations, Electromagnetic Field

\section{Introduction}

Recently, the realization and generalization of fractional calculus has become of great significance in many fields [1] such as determining voltage-current relationship in a non-ideal capacitor, fractal behavior of a metal insulator solution interface, electromagnetic waves, and recently in electrical circuits such as filters [2]-[7], oscillators [8] [9] [10], passive realization [11] [12] and energy-related issues in supercapacitors [13]. Furthermore, applications of fractional calculus have been reported in many areas such as physics [14], nonlinear oscillation of earthquakes [15], and mathematical biology [16]. 
Fractional calculus has higher capability of providing accurate description than integer dynamical systems. In the circuit theory, the fractional-order element with impedance proportional to $s^{\alpha}$ is considered as a generalized element that covers the conventional circuit elements when the order of elements equals to integer [17]. And the fractional-order mutual inductance is the generalized concept of the mutual inductance in the fractional-order domain [18]. Due to the extra fractional-order parameters, more flexibility is added. During the last 10 years, several promising trials have been introduced for the realizations of the fractional-order element and based on different techniques such as chemical reactions [19], fractal shapes [20], and graphene material [21]. Moreover, many finite circuit approximations were suggested to model fractional-order elements [22] [23] [24] [25].

In the framework of fractional calculus, there are lots of literatures about Maxwell's equations. Tarasov [26] formulated the fractional Green's, Stokes' and Gauss's theorems and realized the proofs of these theorems for simple regions. He considered fractional nonlocal Maxwell's equations and the corresponding fractional wave equations. Tarasov [27] used the fractional integrals to describe fractal distribution. Tarasov [28] suggested a fractional generalization of the integral Maxwell equations. Lazo [29] proposed a spatially symmetric and causal gauge invariant fractional electromagnetic field from a Lagrangian formulation. Electromagnetic field propagation has also been modeled using the fractional calculus [30].

It is well known that circuit theory is a limiting special case of electromagnetic field theory. In particular, the characterization of the four classical circuit elements can be given an elegant electromagnetic interpretation by the quasi-static expansion of Max-well's equations [31] [32] [33]. The electromagnetic interpretation of classical circuit elements in Laplace domain is introduced in [34]. The fractional-order elements appeared, but there was no electromagnetic field interpretation of fractional-order elements yet. So, by considering fractional-order time derivatives, this paper is to give an analogous interpretation for the characterization of fractional-order elements.

This paper is organized as follows: in Section 2, the notions of fractional calculus and fractional-order elements and fractional Maxwell's equations are presented. After that, the quasi-static expansion of fractional Maxwell's equations and the electromagnetic field interpretation of fractional-order elements are shown in Section 3. Finally, conclusions are found in Section 4. In this section, some preliminary definitions are presented.

\section{Preliminaries}

This section is devoted to introducing some preliminaries, which help us to state the results of the paper.

\subsection{Fractional Calculus}

The first definition is about fractional derivative operator as a generalization for 
classical derivative operator. There are three kinds of definitions about the fractional derivative, the Riemann-Liouville definition, the Grunwald-Letnikov definition and the Caputo definition. According to the Caputo definition for fractional derivative, the derivative of order $\alpha$ for function $f(t)$ is given by

$$
{ }_{a} D_{t}^{\alpha} f(t)=\frac{1}{\Gamma(1-\alpha)} \int_{0}^{t}(t-\tau)^{-\alpha} f^{\prime}(\tau) \mathrm{d} \tau, \text { for } \quad \alpha \in(0,1)
$$

where ${ }_{a} D_{t}^{\alpha}=\frac{\mathrm{d}^{\alpha}}{\mathrm{d} t^{\alpha}}, \Gamma(\cdot)$ is Euler's Gamma function.

\subsection{Fractional-Order Elements}

The template is used to format your paper and style the text. All margins, column widths, line spaces, and text fonts are prescribed; please do not alter them. You may note peculiarities. For example, the head margin in this template measures proportionately more than is customary. This measurement and others are deliberate, using specifications that anticipate your paper as one part of the entire journals, and not as an independent document. Please do not revise any of the current designations.

If $u(t)$ and $i(t)$ are respectively the voltage and current of a fractional-order capacitor with order $\alpha \in(0,1)$ and the pseudo-capacitance $C_{\alpha}$, then

$$
i(t)=C_{\alpha} \frac{\mathrm{d}^{\alpha} u(t)}{\mathrm{d} t^{\alpha}}
$$

The impedance of a fractional-order capacitor with order $\alpha$ and the pseudo-capacitance $C_{\alpha}$ equals $z(s)=1 / C_{\alpha}(\mathrm{j} \omega)^{\alpha}$ in frequency domain.

Similarly, for $u(t)$ and $i(t)$ as the voltage and current of a fractional-order inductor with order $\beta \in(0,1)$ and the pseudo-inductance $L_{\beta}$, the following relation holds,

$$
u(t)=L_{\beta} \frac{\mathrm{d}^{\beta} i(t)}{\mathrm{d} t^{\beta}}
$$

The impedance of a fractional-order inductor with order $\beta$ and pseudo-inductance $L_{\alpha}$ equals $z(s)=L_{\beta}(\mathrm{j} \omega)^{\beta}$ in frequency domain.

For fractional coupling mutual inductance, we assume the primary and secondary inductors are of different fractional orders $\alpha, \beta$ respectively. Consequently, in this case the mutual inductance is not symmetric, and the mutual inductance could be considered of the fractional-order $\gamma$,

$$
\left\{\begin{array}{l}
u_{1}=L_{11} \frac{\mathrm{d}^{\alpha} i_{1}}{\mathrm{~d} t^{\alpha}}+M_{12} \frac{\mathrm{d}^{\gamma_{12}} i_{2}}{\mathrm{~d} t^{\gamma_{12}}} \\
u_{2}=M_{21} \frac{\mathrm{d}^{\gamma_{21}} i_{1}}{\mathrm{~d} t^{\gamma_{21}}}+L_{22} \frac{\mathrm{d}^{\beta} i_{2}}{\mathrm{~d} t^{\beta}}
\end{array}\right.
$$

In frequency domain, we have,

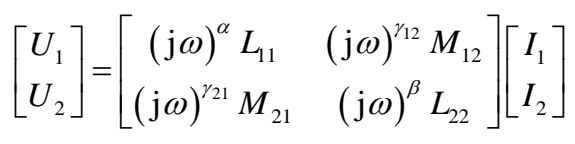


where $\alpha, \beta, \gamma_{12}, \gamma_{21} \in(0,1)$ is the fractional order of primary, secondary inductors and mutual inductance, $L_{11}, L_{22}, M_{12}$ and $M_{21}$ are primary, secondary, mutual inductance. For the traditional case $\alpha, \beta, \gamma_{12}, \gamma_{21}=1$, Equation (5) represents the matrix equation of the integer-order mutual inductor.

\subsection{Fractional Maxwell's Equations}

According to Faraday's law for induction and with a general fractional-order derivative $\alpha$, we assume that $\alpha \in(0,1)$ in this paper, and the parameter $\sigma_{t}$ [35] (auxiliary parameter, introduced to be consistent with the time dimensionality.) which represents the fractional time components in the system, we have the first Maxwell's curl equation [36],

$$
\nabla \times \boldsymbol{E}=-\mu \frac{1}{\sigma_{t}^{1-\alpha}} \frac{\partial^{\alpha} \boldsymbol{H}}{\partial t^{\alpha}}
$$

Similarly, for some general fractional-order derivative $\alpha$, the 2nd Maxwell curl equation can be written as

$$
\nabla \times \boldsymbol{H}=\boldsymbol{J}+\varepsilon \frac{1}{\sigma_{t}^{1-\alpha}} \frac{\partial^{\alpha} \boldsymbol{E}}{\partial t^{\alpha}}
$$

The other two equations are independent of time and stay the same,

$$
\begin{aligned}
\nabla \cdot \boldsymbol{B} & =0 \\
\nabla \cdot \boldsymbol{D} & =\rho
\end{aligned}
$$

\section{Electromagnetic Interpretation of Fractional-Order Elements}

In terms of a quasi-static expansion of fractional Maxwell's equations, the electromagnetic interpretation of the fractional-order elements is presented in this section.

\subsection{Quasi-Static Expansion of Fractional Maxwell's Equations}

Quasi-static approximation is used to simplify Maxwell's equations involving a family of dynamic fields, produced by accelerating charges and time varying currents. For wave velocities less than that of light, these fields can be decomposed into "static" fields for a finite time duration called family time, to avoid complications due to retarded potentials. Family time is defined as, $\tau=a t$ where $a$ is the time-rate parameter. Then we have,

$$
\frac{\partial^{\alpha} \boldsymbol{E}}{\partial t^{\alpha}}=a^{\alpha} \frac{\partial^{\alpha} \boldsymbol{E}}{\partial \tau^{\alpha}}, \frac{\partial^{\alpha} \boldsymbol{H}}{\partial t^{\alpha}}=a^{\alpha} \frac{\partial^{\alpha} \boldsymbol{H}}{\partial \tau^{\alpha}} \text {. Applying the equations to (6) and (7), we }
$$
get,

$$
\begin{gathered}
\nabla \times \boldsymbol{E}=-\mu \frac{a^{\alpha}}{\sigma_{t}^{1-\alpha}} \frac{\partial^{\alpha} \boldsymbol{H}}{\partial \tau^{\alpha}} \\
\nabla \times \boldsymbol{H}=\boldsymbol{J}+\varepsilon \frac{a^{\alpha}}{\sigma_{t}^{1-\alpha}} \frac{\partial^{\alpha} \boldsymbol{E}}{\partial \tau^{\alpha}}
\end{gathered}
$$


Since the electric field and magnetic field are functions of the parameter $a$ as well as functions of $x, y, z$, and $\tau$, the fields can be expressed as a power series in $a^{\alpha}$,

$$
\begin{aligned}
& \boldsymbol{E}=\boldsymbol{E}_{0}+a^{\alpha} \boldsymbol{E}_{1}+\left(a^{\alpha}\right)^{2} \boldsymbol{E}_{2}+\cdots \\
& \boldsymbol{H}=\boldsymbol{H}_{0}+a^{\alpha} \boldsymbol{H}_{1}+\left(a^{\alpha}\right)^{2} \boldsymbol{H}_{2}+\cdots
\end{aligned}
$$

where $\boldsymbol{E}_{k}(x, y, z, \tau)=\frac{1}{k !}\left[\partial^{k} \boldsymbol{E}\left(x, y, z, \tau, a^{\alpha}\right) / \partial\left(a^{\alpha}\right)^{k}\right]_{a^{\alpha}=0}, \quad k=0,1,2, \cdots$, called the $k$ th-order electric field.

And $\boldsymbol{H}_{k}, k=0,1,2, \cdots$, are defined in a similar way, called the $k$ th-order magnetic field, all the other electromagnetic quantities can be expressed, similarly, as power series in $a^{\alpha}$.

Equating coefficients of $\left(a^{\alpha}\right)^{k}$, we get the $k$ th-order fractional Maxwell's equations

For the zero-order set

$$
\begin{aligned}
& \nabla \times \boldsymbol{E}_{0}=0 \\
& \nabla \times \boldsymbol{H}_{0}=\boldsymbol{J}_{0}
\end{aligned}
$$

For the first-order set

$$
\begin{gathered}
\nabla \times \boldsymbol{E}_{1}=-\mu \frac{1}{\sigma_{t}^{1-\alpha}} \frac{\partial^{\alpha} \boldsymbol{H}_{0}}{\partial \tau^{\alpha}} \\
\nabla \times \boldsymbol{H}_{1}=\boldsymbol{J}_{1}+\varepsilon \frac{1}{\sigma_{t}^{1-\alpha}} \frac{\partial^{\alpha} \boldsymbol{E}_{0}}{\partial \tau^{\alpha}}
\end{gathered}
$$

And, in general, for the $k$ th-order set,

$$
\begin{gathered}
\nabla \times \boldsymbol{E}_{k}=-\mu \frac{1}{\sigma_{t}^{1-\alpha}} \frac{\partial^{\alpha} \boldsymbol{H}_{k-1}}{\partial \tau^{\alpha}} \\
\nabla \times \boldsymbol{H}_{k}=\boldsymbol{J}_{k}+\varepsilon \frac{1}{\sigma_{t}^{1-\alpha}} \frac{\partial^{\alpha} \boldsymbol{E}_{k-1}}{\partial \tau^{\alpha}}
\end{gathered}
$$

Assuming transverse electromagnetic waves travelling in an isotropic medium, i.e. electric field along $\mathrm{x}$-axis, magnetic field along $\mathrm{y}$-axis and wave propagation along $\mathrm{z}$-axis, the fractional Maxwell's equations in the transform domain are given as follows,

$$
\begin{gathered}
\mathrm{j} \boldsymbol{k}_{z} \cdot \boldsymbol{E}_{x}=\frac{\rho}{\varepsilon} \\
\mathrm{j} \boldsymbol{k}_{z} \cdot \boldsymbol{H}_{y}=0 \\
\mathrm{j} \boldsymbol{k}_{z} \times \boldsymbol{E}_{x}=-(\mathrm{j} \omega)^{\alpha} \frac{1}{\sigma_{t}^{1-\alpha}} \mu \boldsymbol{H}_{y} \\
\mathrm{j} k_{z} \times \boldsymbol{H}_{y}=\sigma E+(\mathrm{j} \omega)^{\alpha} \frac{1}{\sigma_{t}^{1-\alpha}} \varepsilon \boldsymbol{E}_{x}
\end{gathered}
$$

where $\omega$ is angular frequency, $k$ is propagation vector, $\lambda$ is wavelength, 
$\sigma$ is electrical conductivity and $\rho$ is charge density.

Then we get the zero- and first-order fractional Maxwell's equations,

$$
\begin{gathered}
\mathrm{j} k E_{0}=0 \\
-\mathrm{j} k H_{0}=\sigma E_{0} \\
-\mathrm{j} k E_{1}=-(\mathrm{j} \omega)^{\alpha} \frac{1}{\sigma_{t}^{1-\alpha}} \mu H_{0} \\
-\mathrm{j} k H_{1}=\sigma E_{1}+(\mathrm{j} \omega)^{\alpha} \frac{1}{\sigma_{t}^{1-\alpha}} \varepsilon E_{0}
\end{gathered}
$$

\subsection{An Electromagnetic Interpretation of Fractional-Order Elements}

Consider the characteristic impedance of a fractional electromagnetic system, given by the ratio of the electric and magnetic fields with a factor of " $j$ " since the fields are orthogonal,

$$
Z=\frac{\mathrm{j} E}{H}=\frac{\mathrm{j}\left(E_{0}+E_{1}\right)}{\left(H_{0}+H_{1}\right)}
$$

Then, a resistor is identified to be a fractional electromagnetic system whose first-order fields are negligible compared to its zero-order fields. i.e. $E_{1}, H_{1}=0$.

$$
Z=\frac{\mathrm{j} E_{0}}{H_{0}}=\frac{k}{\sigma}=\frac{2 \pi}{\sigma \lambda}
$$

The characteristic impedance is a resistor.

A fractional-order inductor is identified to be a fractional electromagnetic system which only considers the first order electric field and the zero-order magnetic field i.e. $E_{0}, H_{1}=0$.

$$
Z=\frac{\mathrm{j} E_{1}}{H_{0}}=\frac{\mathrm{j} E_{1}}{H_{0}}=\frac{(\mathrm{j} \omega)^{\alpha} \mu \lambda}{\sigma_{t}^{1-\alpha} 2 \pi}
$$

The characteristic impedance is a fractional-order inductor. As $\alpha=1$, the system reduces to an inductor.

A fractional-order capacitor is identified to be a fractional electromagnetic system where only consider the first order magnetic field and the zero-order electric field i.e. $E_{1}, H_{0}=0$.

$$
Z=\frac{\mathrm{j} E_{0}}{H_{1}}=\frac{\mathrm{j} E_{0}}{H_{1}}=\frac{\sigma_{t}^{1-\alpha} 2 \pi}{(j \omega)^{\alpha} \varepsilon \lambda}
$$

The characteristic impedance is a fractional-order capacitor. As $\alpha=1$, the system reduces to a capacitor.

The fractional-order mutual inductor can also be seen as a fractional electromagnetic system. We assume that, for convenience, $\alpha, \beta$ is the order of primary and secondary inductor. What we care about is the effect of the magnetic field on the electric field in fractional mutual coupled circuits, from (10), we obtain,

In primary inductor, 


$$
\nabla \times \boldsymbol{E}_{t 1}=-\mu_{1} \frac{a^{\alpha}}{\sigma_{t}^{1-\alpha}} \frac{\partial^{\alpha} \boldsymbol{H}_{t 1}}{\partial \tau^{\alpha}}
$$

In secondary inductor,

$$
\nabla \times \boldsymbol{E}_{t 2}=-\mu_{2} \frac{a^{\beta}}{\sigma_{t}^{1-\beta}} \frac{\partial^{\beta} \boldsymbol{H}_{t 2}}{\partial \tau^{\beta}}
$$

where $\boldsymbol{E}_{t 1}, \boldsymbol{H}_{t 1}, \boldsymbol{E}_{t 2}, \boldsymbol{H}_{t 2}$ is the total fields in primary and secondary inductor.

As the order of primary inductor is different from that of the secondary inductor, $H_{t 1}, H_{t 2}$ can be regarded as the superposition of the magnetic fields in linear system, we assume that,

$$
\begin{gathered}
\boldsymbol{H}_{t 1}=l_{11} \boldsymbol{H}_{c 1}+l_{12} \frac{1}{\sigma_{t}^{\alpha-\gamma_{12}}} \frac{\partial^{\gamma_{12}-\alpha}}{\partial \tau^{\gamma_{12}-\alpha}} \boldsymbol{H}_{c 2} \\
\boldsymbol{H}_{t 2}=l_{21} \frac{1}{\sigma_{t}^{\beta-\gamma_{21}}} \frac{\partial^{\gamma_{21}-\beta}}{\partial \tau^{\gamma_{21}-\beta}} \boldsymbol{H}_{c 1}+l_{12} \boldsymbol{H}_{c 2}
\end{gathered}
$$

where $\boldsymbol{H}_{c 1}, \boldsymbol{H}_{c 2}$ is the fields produced by primary and secondary inductor, $l_{11}, l_{12}, l_{21}, l_{22}$, functions of the space coordinates alone, represent the contribution to the total magnetic field from $\boldsymbol{H}_{c 1}$ or $\boldsymbol{H}_{c 2}$, and $\gamma_{12}, \gamma_{21}$ is the order of mutual inductance in primary and secondary inductor, respectively. And when $\alpha=\beta$, we have $\gamma_{12}=\gamma_{21}=\alpha$.

Substituting (34) (35) into (32) (33) respectively, we get,

$$
\begin{gathered}
\nabla \times \boldsymbol{E}_{t 1}=-\mu_{1} \frac{a^{\alpha}}{\sigma_{t}^{1-\alpha}} \frac{\partial^{\alpha}}{\partial \tau^{\alpha}} l_{11} \boldsymbol{H}_{c 1}-\mu_{1} \frac{a^{\alpha}}{\sigma_{t}^{1-\gamma_{12}}} \frac{\partial^{\gamma_{12}}}{\partial \tau^{\gamma_{12}}} l_{12} \boldsymbol{H}_{c 2} \\
\nabla \times \boldsymbol{E}_{t 2}=-\mu_{2} \frac{a^{\beta}}{\sigma_{t}^{1-\gamma_{21}}} \frac{\partial^{\gamma_{21}}}{\partial \tau^{\gamma_{21}}} l_{12} \boldsymbol{H}_{c 1}-\mu_{2} \frac{a^{\beta}}{\sigma_{t}^{1-\beta}} \frac{\partial^{\beta}}{\partial \tau^{\beta}} l_{22} \boldsymbol{H}_{c 2}
\end{gathered}
$$

By expressing the fields in primary inductor, $\boldsymbol{E}_{t 1}, \boldsymbol{H}_{c 1}, \boldsymbol{H}_{c 2}$, as power series in $a^{\alpha}$, and equating coefficients of $\left(a^{\alpha}\right)^{k}$, the corresponding first-order fractional Maxwell's equation of the primary inductor can be deduced as we get (26). In the transform domain, we have

$$
-\mathrm{j} k_{1} E_{t 11}=-(\mathrm{j} \omega)^{\alpha} \frac{1}{\sigma_{t}^{1-\alpha}} \mu_{1} H_{t 01}
$$

where $E_{t 11}=\left.\frac{\partial E_{t 1}}{\partial a^{\alpha}}\right|_{a^{\alpha}=0}$ is the total first order electric field in primary inductor, and $H_{t 01}=\left.l_{11} H_{c 1}\right|_{a^{\alpha}=0}+\left.\frac{1}{\sigma_{t}^{\alpha-\gamma_{12}}}(j \omega)^{\gamma_{12}-\alpha} l_{12} H_{c 2}\right|_{a^{\alpha}=0}$, the total zero-order magnetic field in primary inductor.

In the similar way, we can get the corresponding equation in secondary inductor,

$$
-\mathrm{j} k_{2} E_{t 12}=-(\mathrm{j} \omega)^{\beta} \frac{1}{\sigma_{t}^{1-\beta}} \mu_{2} H_{t 02}
$$

where $E_{t 12}=\left.\frac{\partial E_{t 2}}{\partial a^{\beta}}\right|_{a^{\beta}=0}$ is the first order electric field in secondary inductor, and 
$H_{t 02}=\left.\frac{1}{\sigma_{t}^{\beta-\gamma_{21}}}(j \omega)^{\gamma_{21}-\beta} l_{21} H_{c 1}\right|_{a^{\beta}=0}+\left.l_{22} H_{c 2}\right|_{a^{\beta}=0}$, the total zero-order magnetic field in secondary inductor.

As the first term of the power series expansion of magnetic field in $a^{\alpha}$ is the same as the first term of the power series expansion of magnetic field in $a^{\beta}$, let $H_{c 01}=\left.H_{c 1}\right|_{a^{\alpha}=0}=\left.H_{c 1}\right|_{a^{\beta}=0}$, and $H_{c 02}=\left.H_{c 2}\right|_{a^{\alpha}=0}=\left.H_{c 2}\right|_{a^{\beta}=0}$.

Then we obtain,

$$
\begin{gathered}
-\mathrm{j} k_{1} E_{t 11}=-\mu_{1} \frac{1}{\sigma_{t}^{1-\alpha}}(\mathrm{j} \omega)^{\alpha} l_{11} H_{c 01}-\mu_{1} \frac{1}{\sigma_{t}^{1-\gamma_{12}}}(\mathrm{j} \omega)^{\gamma_{12}} l_{12} H_{c 02} \\
-\mathrm{j} k_{2} E_{t 12}=-\mu_{2} \frac{1}{\sigma_{t}^{1-\gamma_{21}}}(\mathrm{j} \omega)^{\gamma_{21}} l_{21} H_{c 01}-\mu_{2} \frac{1}{\sigma_{t}^{1-\beta}}(\mathrm{j} \omega)^{\beta} l_{22} H_{c 02}
\end{gathered}
$$

when $H_{c 02}=0$,

$$
\begin{array}{r}
z_{11}=\frac{\mathrm{j} E_{t 11}}{H_{c 01}}=\frac{k_{1}}{\sigma_{t}^{1-\alpha}}(\mathrm{j} \omega)^{\alpha} l_{11} \mu_{1}=(\mathrm{j} \omega)^{\alpha} l_{11} \mu_{1} \lambda_{1} /\left(\sigma_{t}^{1-\alpha} 2 \pi\right) \\
z_{21}=\frac{\mathrm{j} E_{t 12}}{H_{c 01}}=(\mathrm{j} \omega)^{\gamma_{21}} l_{21} \mu_{2} \frac{k_{2}}{\sigma_{t}^{1-\gamma_{21}}}=(\mathrm{j} \omega)^{\gamma_{21}} l_{21} \mu_{2} \lambda_{2} /\left(\sigma_{t}^{1-\gamma_{21}} 2 \pi\right)
\end{array}
$$

when $H_{c 01}=0$,

$$
\begin{gathered}
z_{22}=\frac{\mathrm{j} E_{t 12}}{H_{c 02}}=(\mathrm{j} \omega)^{\beta} l_{22} \mu_{2} \frac{k_{2}}{\sigma_{t}^{1-\beta}}=(\mathrm{j} \omega)^{\beta} l_{22} \mu_{2} \lambda_{2} /\left(\sigma_{t}^{1-\beta} 2 \pi\right) \\
z_{12}=\frac{\mathrm{j} E_{t 11}}{H_{c 02}}=(\mathrm{j} \omega)^{\gamma_{12}} l_{12} \mu_{1} \frac{k_{1}}{\sigma_{t}^{1-\gamma_{12}}}=(\mathrm{j} \omega)^{\gamma_{12}} l_{12} \mu_{1} \lambda_{1} /\left(\sigma_{t}^{1-\gamma_{12}} 2 \pi\right)
\end{gathered}
$$

where $z_{11}, z_{12}, z_{21}, z_{22}$ represent the element of characteristic impedance matrix.

Then we have the characteristic impedance matrix of the fractional electromagnetic system,

$$
\boldsymbol{Z}=\left[\begin{array}{ll}
z_{11} & z_{12} \\
z_{21} & z_{22}
\end{array}\right]=\left[\begin{array}{cc}
(\mathrm{j} \omega)^{\alpha} l_{11} \mu_{1} \lambda_{1} /\left(\sigma_{t}^{1-\alpha} 2 \pi\right) & (\mathrm{j} \omega)^{\gamma_{12}} l_{12} \mu_{1} \lambda_{1} /\left(\sigma_{t}^{1-\gamma_{12}} 2 \pi\right) \\
(\mathrm{j} \omega)^{\gamma_{21}} l_{21} \mu_{2} \lambda_{2} /\left(\sigma_{t}^{1-\gamma_{21}} 2 \pi\right) & (\mathrm{j} \omega)^{\beta} l_{22} \mu_{2} \lambda_{2} /\left(\sigma_{t}^{1-\beta} 2 \pi\right)
\end{array}\right]
$$

The characteristic impedance matrix is a fractional-order mutual inductor. As the element order equal to 1 , i.e. $\alpha=\beta=\gamma_{12}=\gamma_{21}=1$, the fractional-order mutual inductor reduce to the mutual inductor.

\section{Concluding Remarks}

In terms of quasi-static expansion of the fractional Maxwell's equations considering fractional-order time derivatives, we get the electromagnetic interpretation of several fractional-order elements: fractional-order inductor, fractional-order capacitor, and fractional-order mutual inductance. It shows that fractional-order elements can also be interpreted as a fractional electromagnetic system. As the element order equal to 1 , the interpretation of fractional-order elements matches that of the classical circuit elements: $L, C$, and the mutual inductance, respectively.

Future research will aim to get the electromagnetic interpretation of fractional-order memristor fractional high-order elements. 


\section{Acknowledgements}

The authors wish to thank Prof. Liang, for giving several suggestions which are included in the present revision. The authors are also grateful to Dr. Ma and M. S. Qi for their very helpful comments and suggestions.

\section{References}

[1] Elwakil, A.S. (2010) IEEE Circuits \& Systems Magazine, 10, 40-50. https://doi.org/10.1109/MCAS.2010.938637

[2] Radwan, A.G. and Salama, K.N. (2011) IEEE Transactions on Circuits and Systems I: Regular Papers, 58, 2388-2397.

[3] Radwan, A.G. and Salama, K.N. (2012) Circuits, Systems, and Signal Processing, 31, 1901-1915. https://doi.org/10.1007/s00034-012-9432-Z

[4] Moaddy, K., Radwan, A.G., Salama, K.N., Momani, S. and Hashim, I. (2012) Computers \& Mathematics with Applications, 64, 3329-3339. https://doi.org/10.1016/j.camwa.2012.01.005

[5] Magin, R.L. (2004) Critical Reviews in Biomedical Engineering, 32, 1. https://doi.org/10.1615/CritRevBiomedEng.v32.10

[6] Radwan, A.G., Moddy, K. and Momani, S. (2011) Computers \& Mathematics with Applications, 62, 961-970. https://doi.org/10.1016/j.camwa.2011.04.047

[7] Radwan, A.G., Moaddy, K., Salama, K.N., Momani, S. and Hashim, I. (2013) Control and Switching Synchronization of Fractional Order Chaotic Systems Using Active Control Technique. Journal of Advanced Research, 5, 125-132.

[8] Radwan, A.G., Soliman, A.M., Elwakil, A.S. and Sedeek, A. (2009) Chaos, Solitons \& Fractals, 40, 2317-2328. https://doi.org/10.1016/j.chaos.2007.10.033

[9] Radwan, A.G. (2012) Journal of Fractional Calculus and Applications, 3, 1-15.

[10] Chen, Y.Q., Ahn, H.S. and Podlubny, I. (2006) Signal Processing, 86, 2611-2618. https://doi.org/10.1016/j.sigpro.2006.02.011

[11] Sarafraz, M.S. and Tavazoei, M.S. (2015) IEEE Transactions on Circuits and Systems I: Regular Papers, 62, 2829-2835. https://doi.org/10.1109/TCSI.2015.2482340

[12] Sarafraz, M.S. and Tavazoei, M.S. (2017) IEEE Transactions on Circuits and Systems I: Regular Papers, 64, 585-595. https://doi.org/10.1109/TCSI.2016.2614249

[13] Elwakil, A.S., Allagui, A., Freeborn, T.J. and Maundy, B.J. (2017) AEU-International Journal of Electronics and Communications, 78, 209-212. https://doi.org/10.1016/j.aeue.2017.03.027

[14] Alikhani, R. and Bahrami, F. (2013) Global Solutions for Nonlinear Fuzzy Fractional Integral and Integrodifferential Equations. Communications in Nonlinear Science and Numerical Simulation, 18, 2007-2017.

[15] Fouda, M.E. and Radwan, A.G. (2013) Journal of Fractional Calculus and Applications, 4, 1-7.

[16] Shamim, A., Radwan, A.G. and Salama, K.N. (2011) IEEE Microwave and Wireless Components Letters, 21, 117-119. https://doi.org/10.1109/LMWC.2010.2098861

[17] Westerlund, S. and Ekstam, L. (1994) IEEE Transactions on Dielectrics and Electrical Insulation, 1, 826-838.

[18] Soltan, A., Radwan, A.G. and Soliman, A.M. (2016) International Journal of Circuit Theory and Applications, 44, 85. 
[19] Biswas, K., Sen, S. and Dutta, P.K. (2006) Sensors \& Actuators B Chemical, 119, 186-191. https://doi.org/10.1016/j.snb.2005.12.011

[20] Cisse Haba, T., Ablart, G., Camps, T. and Olivie, F. (2005) Chaos, Solitons \& Fractals, 24, 479-490. https://doi.org/10.1016/j.chaos.2003.12.095

[21] Elshurafa, A.M., Almadhoun, M.N., Salama, K.N. and Alshareef, H.N. (2013) Applied Physics Letters, 102, Article ID: 232901. https://doi.org/10.1063/1.4809817

[22] Krishna, B.T. and Reddy, K.V.V.S. (2008) Active and Passive Electronic Components, 2008, Article ID: 369421. https://doi.org/10.1155/2008/369421

[23] Nakagawa, M. and Sorimachi, K. (1992) IEICE Transactions on Fundamentals of Electronics, Communications and Computer Sciences, 75, 1814-1819.

[24] Sugi, M., Hirano, Y., Miura, Y.F. and Saito, K. (1999) IEICE Transactions on Fundamentals of Electronics, Communications and Computer Sciences, 82, 1627-1635.

[25] Sugi, M., Hirano, Y., Miura, Y.F. and Saito, K. (2002) Colloids and Surfaces A: Physicochemical and Engineering Aspects, 198, 683-688. https://doi.org/10.1016/S0927-7757(01)00988-8

[26] Tarasov, V.E. (2008) Annals of Physics, 323, 2756-2778. https://doi.org/10.1016/j.aop.2008.04.005

[27] Tarasov, V.E. (2005) Physics of Plasmas, 12, 461-307. https://doi.org/10.1063/1.1994787

[28] Tarasov, V.E. (2008) Modern Physics Letters A, 21, 1587-1600. https://doi.org/10.1142/S0217732306020974

[29] Lazo, M.J. (2011) Physics Letters A, 375, 3541-3546. https://doi.org/10.1016/j.physleta.2011.08.033

[30] Bia, P., Mescia, L. and Caratelli, D. (2016) Mathematical Problems in Engineering, 2016, Article ID: 5676903.

[31] Fano, R.M., Chu, L.J. and Adler, R.B. (1960) Electromagnetic Fields, Energy, and Forces. Wiley, New York, Ch. 6.

[32] Penfield, P. and Rafuse, R.P. (1962) Varactor Applications. Solid-State Circuits Conference. Digest of Technical Papers. 1961 IEEE International, Vol. 4, 95-95.

[33] Chua, L.O. (1971) IEEE Transactions on Circuit Theory, 18, 507-519. https://doi.org/10.1109/TCT.1971.1083337

[34] Kubendran, R. (2012) Electromagnetic and Laplace Domain Analysis of Memristance and Associative Learning using Memristive Synapses Modeled in SPICE. International Conference on Devices, Circuits and Systems, Coimbatore, 15-16 March 2012, 622-626. https://doi.org/10.1109/ICDCSyst.2012.6188646

[35] Gómezaguilar, J.F., Rosalesgarcía, J.J., Bernalalvarado, J.J. and Córdovafraga, T. (2012) Revista Mexicana De Física, 58, 348-352.

[36] Ismail, R. and Radwan, A.G. (2012) Rectangular Waveguides in the Fractional-Order Domain. International Conference on Engineering and Technology, 2-6 November 2012, 1-6. https://doi.org/10.1109/ICEngTechnol.2012.6396151 